\title{
Large Reduction in Volume with the Intensive Treatment of Lymphedema: Reduction of Fluids?
}

\author{
Jose Maria Pereira de Godoy, MD, $\mathrm{PhD}^{1}$ Isabella Pereira Gonçalves, $\mathrm{PhT}^{2}$ Stemarys Barufi, $\mathrm{PhT}^{2}$ \\ Maria de Fátima Guerreiro Godoy, OT, $\mathrm{PhD}^{2}$
}

${ }^{1}$ Department of Cardiology and Cardiovascular Surgery, Medicine School, São José do Rio Preto (FAMERP); CNPq (National Council for Research and Development), Brazil

2 Lymphovenous Rehabilitation, Medicine School, São Jose do Rio Preto

Address for correspondence and reprint requests José Maria Pereira de Godoy, MD, PhD, Rua Floriano Peixoto, 2950, São José do Rio Preto, Sao Paulo, Brazil (e-mail: godoyjmp@riopreto.com.br).

(FAMERP); Godoy Clinic, Brazil

Int J Angiol 2012;21:171-174.

\begin{abstract}
The aim of this study is to report on the intensive treatment of lymphedema of the legs demonstrating a large reduction in volume in a short period of time. The case of a 29-year-old female patient, who developed the most serious form of lymphedema, associated with verrucosities of the leg and genitalia is reported. Elephantiasis evolved after surgery and radiotherapy. The patient was treated at the Clinica Godoy in Sao Jose do Rio Preto, Brazil using an intensive course of treatment. Baseline and then daily evaluations of the leg perimeter and body weight were performed during treatment. Intensive treatment for 8 hours daily was performed on an outpatient basis using manual and mechanical (RAGodoy device, São Jose do Rio Preto, São Paulo-Brazil) lymph drainage and the continuous use of a compression garment made of a cotton-polyester textile and adjusted every 3 hours. A reduction of $31 \mathrm{~kg}$ was seen in 10 days (over 2 weeks); in the first few days, the patient lost $6 \mathrm{~kg}$ per day. Due to the excess of skin, the length of intensive treatment sessions was reduced to avoid the compression garment causing a tourniquet effect, the size of the compression stocking was only adjusted once

\section{Keywords}

- elephantiasis

- treatment

- intensive per day and daily walks were included in the treatment program. After 1 month, the size of the leg was reduced by another $4 \mathrm{~kg}$. Intensive lymphedema treatment is an option that rapidly reduces edema, and constant use of low-stretch compression maintains the result achieved and continues to reduce the swelling.
\end{abstract}

Lymphedema usually affects the poor, there is no cure and there are few therapeutic perspectives in the private sector. This is exacerbated in less developed countries, where lack of government resources and skilled health workers leads to marginalization of the disease.

A combination of different therapies is recommended for the treatment of lymphedema including manual lymph drainage, compression mechanisms, exercise, and hygienic care. ${ }^{1,2}$

In recent years, new options such as mechanical lymph drainage with devices that use passive and active muscle activities, pressure therapy, daily life activities, and nutritional and psychological management have been added. ${ }^{1,3,4}$
Intensive forms of lymphedema treatment have been described with the prospect of rapid control of the edema and maintenance of the treatment outcome. ${ }^{5}$ The purpose of this study is to describe the evolution of this form of treatment with large reductions over a short period of time and a continual reduction in edema on an outpatient treatment basis.

\section{Case Report}

The case of lower limb lymphedema in a 29-year-old female patient is reported. Lymphedema began at the age of 12 years old after the patient was submitted to an exploratory 10.1055/s-0032-1325167. ISSN 1061-1711. 
laparotomy; the initial hypothesis was appendicitis but a lymphoma was discovered.

Although the patient was submitted to sessions of radiotherapy and chemotherapy, she does not remember how many because she was young at that time and her mother has died. The swelling started in the thigh area and then spread to the feet. At first the edema reduced on resting but over the years this ceased to occur.

The patient sought treatment and sessions of lymph drainage, and pressure therapy and the use of elastic compression stockings were also indicated. She started using pressure therapy and elastic stockings every day at her home. The edema worsened and fibrosis developed in the region of the abdomen so she abandoned the treatment. Over time, the swelling got worse and verrucosities began to appear in the leg and genitalia region. She said that there was a constant secretion from the verrucosities.

She was referred to the Clinica Godoy, was weighed, and the perimetry of the leg was measured as it was impossible to perform volumetry due to the size of the leg (-Fig. 1A, B). Intensive therapy was proposed: this included mechanical lymph drainage using the RAGodoy apparatus (7 hours daily), manual lymph drainage using the Godoy technique

hour/day), and a compression garment using a low-stretch stocking $(<50 \%)$ made of a cotton-polyester textile adapted for major deformities (24 hours daily). The compression stocking was adjusted every 3 hours depending on the reduction in volume. There was daily weight loss. On 1 day the loss was $\sim 6 \mathrm{~kg}$ and in 2 weeks, that is 10 days of treatment, the patient lost $31 \mathrm{~kg}$. The following week she lost another $6 \mathrm{~kg}$ totaling $37 \mathrm{~kg}$ in 3 weeks.

After the fifth day of intensive treatment, the patient began to do daily walks with adjustments to the compression garment being made before and after. After the third week, the patient returned to her home where she was submitted to mechanical lymph drainage for 2 hours per day and a 1-hour walk with the stocking being adjusted as necessary. At this stage, the low-stretch compression stocking was worn for the

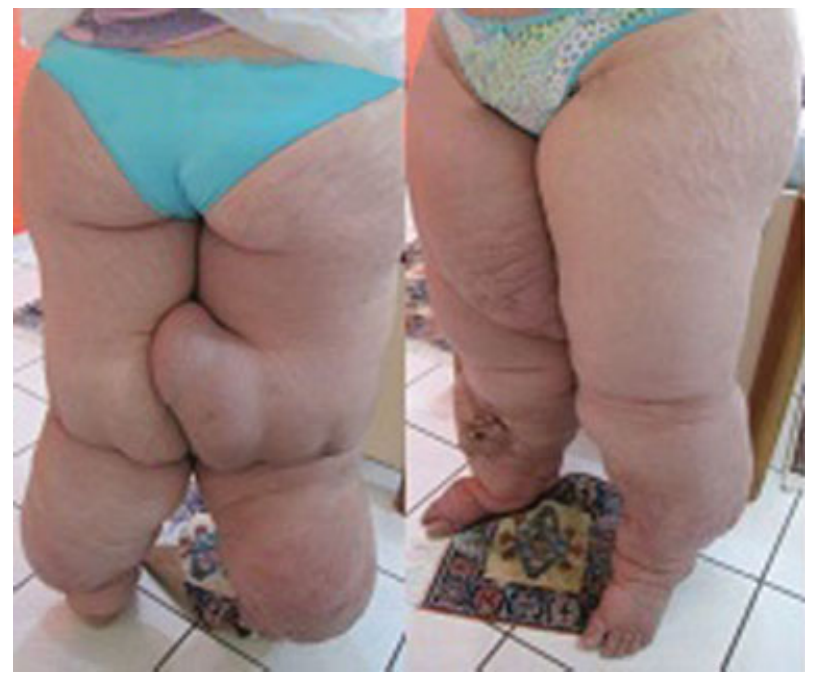

Fig. 1 ( $A$ and $B$ ) Before treatment.

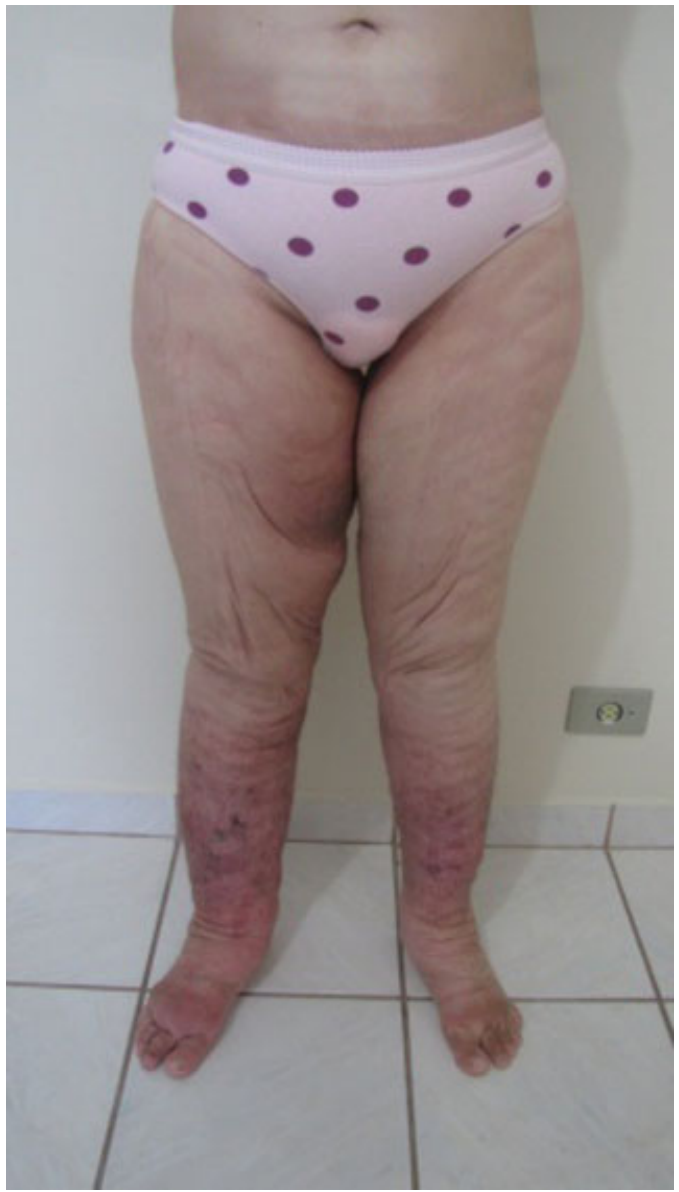

Fig. 2 After 4 weeks of treatment.

entire day (24 hours) associated to an elastic compression stocking. She returned to the clinic 1 month later with a reduction of more than $4 \mathrm{~kg}$, totaling $41 \mathrm{~kg}$ during the intensive treatment program (-Fig. 2). Treatment was continued and evaluations were performed monthly until near total reduction of edema ( $\mathbf{- F i g . 3}$ ). The maintenance phase began after the total reduction of edema.

\section{Discussion}

This case report illustrates intensive outpatient treatment of lymphedema which allowed a large reduction in volume over a short period of time. The results of this treatment program raise the question about the formation of lymphedema. This patient is well educated but even so she evolved with the most severe form of the disease. This shows the difficulties encountered to find an appropriate form of treatment for lymphedema.

Another key aspect of this therapeutic approach is that maintenance of the results continued to reduce the volume of the limb. She learned how to treat and control edema using a form of maintenance that was inexpensive and that gave her independence in respect to treatment.

Mechanical lymph drainage associated with a low-stretch compression garment allows large reductions over a short period of time; in this case, as much as $6 \mathrm{~kg}$ were lost in 1 day 


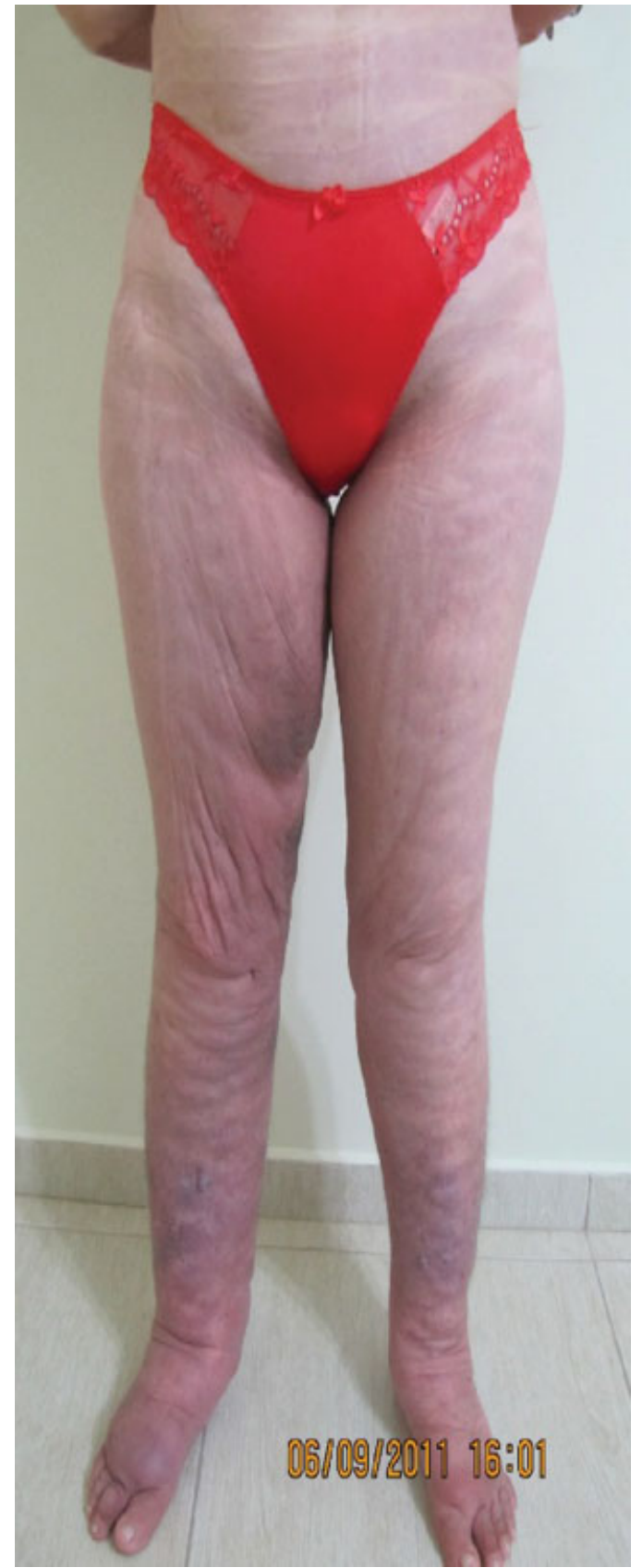

Fig. 3 After 8 weeks of treatment.

at the beginning of the treatment and $31 \mathrm{~kg}$ over 10 days of intensive treatment in 2 weeks.

The quick weight loss in a short period of time raises questions about the constituents of lymphedema where the accumulation of fluid appears to be a major factor. However, other components appear to have been mobilized during treatment. After the treatment, the weights and parameters of the patient's legs were considered normal. Thus, these substances needed to be mobilized to explain the return to a size within the normal range.

As the low-stretch stocking used hooks and eyes for adjustment, compression can be adjusted every hour or as needed. With this approach, adjustment is essential and is achieved without undressing the stocking, which is the main difference between this mechanism and conventional bandages. The compression stocking was worn 24 hours per day to keep the loss that had been attained with mechanical lymph drainage. Studies evaluating cotton-polyester stockings during walking in patients with leg lymphedema confirmed the synergistic effect of compression only when the stockings were well adjusted (phase of publication).

Another interesting aspect was the increase in diuresis; this patient needed to urinate at $\sim 50$-minute intervals during the day; the number of times dropped at night but it was still frequent. The loss of large volumes per day shows that the liquid component in lymphedema is very high and that part of the excess weight can be eliminated by diuresis. However, the proteins and macromolecules are carried in the blood stream and redistributed throughout the body. Thus, reduction of the lymphedema is possible.

The need for constant adjustment of the low-stretch compression is related to its mechanism of action; the muscle activity generates a working pressure with peaks and valleys in pressure at the skin-stocking interface. Thus, the reduction in limb size gradually leads to a reduction in the effect of the working pressure. Compression stockings exert resting and working pressures, where the working pressure depends on the resting pressure exerted by the stocking. As the stocking loses its elasticity, the working pressure will decrease to zero.

Another aspect to be discussed is that Foldi's concept of reduction of phase I swelling is misleading and outdated. Currently, new approaches allow total or near to total reduction in the first phase of treatment. Thus, phase II of treatment should be initiated after the total reduction of edema. This new concept is important because it gives the therapist the opportunity to provide a better outcome for patients. In the case reported here, the intensity of treatment was reduced to allow for skin retraction. Thus, just with clinical treatment and without surgery, it is possible to leave the limb within the normal size range.

Another aspect to be considered in this case is the pathophysiology of lymphedema of the patient. She has a history of surgery and radiotherapy; however, it is difficult to rule out the possibility of an association with late primary lymphedema. But regardless of the cause of lymphedema, it is possible to reduce the swelling to normal or near-normal leg sizes. Therefore, this therapeutic approach is useful for most types of lymphedema.

The most important aspect of this form of therapy is that patients learn to treat their disease on their own and have almost complete independence and manage to keep the limb within the normal size range. The medical team needs to provide guidance on preventing infection and treatment when present. Occupational activities together with compression are the basis for maintenance and so the supervision by an occupational therapist is crucial in this form of therapy.

\section{Conclusion}

Intensive treatment of lymphedema is an option which provides rapid reduction in the swelling, and low-stretch 
174 Intensive treatment of lymphedema Pereira de Godoy et al.

compression maintains the result achieved and continues to reduce the limb size.

\section{References}

1 de Godoy JM, de Godoy MdeF. Godoy \& Godoy technique in the treatment of lymphedema for under-privileged populations. Int J Med Sci 2010;7(2):68-71

2 Kerchner K, Fleischer A, Yosipovitch G. Lower extremity lymphedema update: pathophysiology, diagnosis, and treatment guidelines. J Am Acad Dermatol 2008;59(2):324-331 Review
3 Pereira de Godoy JM, da Silva SH, Guerreiro Godoy MdeF. Interference of the surgical treatment of breast cancer on personal hygiene. Breast J 2008;14(6):607

4 de Godoy JM, Godoy Mde F. Development and evaluation of a new apparatus for lymph drainage: preliminary results. Lymphology 2004;37(2):62-64

5 Pereira de Godoy JM, Azoubel LM, de Fátima Guerreiro de Godoy M. Intensive treatment of leg lymphedema. Indian J Dermatol 2010;55(2):144-147 